\title{
Home Visit Safety: Preventing the Frights; Research is Needed
}

\author{
James Siberski, MS, CMC
}

Department of Gerontology, Misericordia University, USA *Corresponding author: James Siberski, MS, CMC, Assistant Professor, Department of Gerontology,
Misericordia University, Pennsylvania, USA

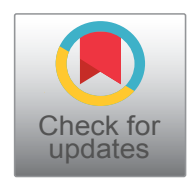

This author was doing a home visit behavioral assessment on a 33-year-old ID male. During the assessment the author reminded him that he had an appointment with the dentist the next day. To which he replied "hey, hey don't put the frights in me". The editorial is not intended to put the frights in anyone, rather it is intended to raise the awareness of professionals (nurses, mobile therapists, educational specialists, etc.) who provide services to the ID population of the additional risks that they can encounter once they leave their offices or places of employment. A safe home visit occurs when: There is no violence or threats of violence; the provider does not contract a communicable disease; the provider's car is not damaged; there is no damage to the provider's property; and nothing unwanted e.g. fleas, is taken home.

There are few guidelines for professionals who make home visits to individuals with intellectual disabilities (ID). Professionals have the potential to make many home visits in a single week, yet alone over years. Nonetheless, few if any published safety guidelines exist that focus on the ID client. Visits to the homes of ID clients present numerous out of the ordinary challenges for the professional that can affect safety and wellbeing. Assessment becomes imperative if safety is to be maintained. This author has published on home visit safety and those who would appreciate a general over view are encouraged to read Siberski J (2003) Home Visit Safety Assessment. Social Work Today. September 2003.

Once the referral is received, prior to the visit, it would be beneficial for the provider to send an "Information Booklet" to the client/family. In a simple and concrete manner, this booklet should explain: The mission of the provider; the consumer's expectation of the professional provider; the provider's expectation, e.g., a clean, therapeutic and safe environment; no firearms on the premises, no aggressive animals, reptiles etc.; and the consequences of not adhering to the expectations. The booklet gives the provider a reference point from which to operate if or when issues surface.

Assessment can be accomplished prior to the first visit by reviewing the ID" $S$ client's history, all medical and psychiatric diagnoses, learning disabilities, behavioral issue, etc., in preparation for the first visit. The client with ID may present with unique problems, such as being cognitively/sensory compromised for any number of reasons due to functional $I Q$, depression or other psychiatric issues, medication issues or depending on the age of the client, normal age-related changes. The provider should remember that individuals with ID, even mild ID, handle stress less efficiently than individuals without ID and have a limited repertoire of adaptive coping abilities. All of which can lead to maladaptive behavior that does not meet the definition of a safe visit. The provider initially prepares by dressing appropriately; no cuffed pants as insects can be carried out of infested environments; and no briefcases or bags of value that are not necessary. Carry a charged cell phone for emergencies in order to call for help if being followed on foot or by a vehicle or to check in with the office so that someone knows your whereabouts. If working privately, leave messages on an answering machine noting your time and location as an aid to family or law enforcement if needed. Programming the cell phone with an emergency number so that contact can be made quickly by pressing one button is also helpful. 
Keep your vehicle in working order and full of gas. Have items such as rubber gloves, antiseptic wipes, and a face mask readily available as clients may have a communicable disease or an open area on the body in need of first aid. The provider should always wipe foreign substances from their hands.

Assess the outside environment. Many dangers that could harm the provider, such as exposed wiring, could be present in the client's home. Loose or broken steps or railings and poor outside lighting could cause a fall. Unrestrained animals belonging to the client, neighbors and even strays could be present. Even in well-kept homes, slippery floors and stairs are a hazard. Upon arrival, if the door is generally kept unlocked, check to see if any unauthorized personnel could be in the house.

The inside environment also needs to be addressed carefully. If the client is older or if family caretakers are older which is the case many times since Individuals with ID are living much longer than in the past and their caretakers can be very old, normal age-related changes reduce sensitivity to smells and undetected furnace fumes could be an issue for both client and provider. Assess for the presence of guns and request that they be removed from the premises. If the client is cognitively compromised, all guns, including toys, must be removed as they could be mistaken for real guns by law enforcement and result in a disaster. Does the client hoard paper, cans, etc. rendering the environment unsafe? Could uneaten or unsealed chips, peanuts, cookies or food invite rodents? In addition, always assess for what could be used as a weapon. Are there animals in the house that are inherently dangerous or that could be dangerous? Are throw rugs or floor and stair coverings loose, damaged or worn-out and dangerous? In short, perform a complete assessment of the inside of the house, including the basement, attic and floors in between. (On one occasion, this provider discovered an explosive device in the basement that the father had previously stored).

Assess the family members who will be present. What are the parents' cognitive abilities and is it a problem or concern for you? If the parents are very old, they could have a neurocognitive disorder or per- haps experience delusions and may think that you are trying to take their child and attempt to correct the situation. Because of their disabilities, does the client allow anyone to enter the house? Unidentified personnel should not be present when you make a visit.

On each visit assess the client's cognitive, emotional and behavioral condition upon entering. Does your client have some type of neurocognitive disorder? Have they or a family member been drinking? Is the client disinhibited (frontal lobe issues) and exhibiting dangerous or upsetting behaviors including sexual advances? Agitation, aggression, and irritability are just some behaviors that one can encounter when working with an ID client with a cognitive impairment, psychiatric disorder or medical issue such as delirium. Assess for psychiatric aspects of the client. Although psychiatric conditions such as schizophrenia, depression (agitated), mania, personality disorders and behavioral disorders do not suggest that the client will engage in behavior that could cause harm, it may increase the possibility of harmful behavior. Clients with ID can experience delirium and psychiatric disorders such as delusions, hallucinations and illusions among many other symptoms. The provider must assess clients carefully in order to prevent harm to themselves. The ID client who is delirious and hears the slamming of a door and misidentifies it as gunfire (illusion) may attempt self-defense and injure the provider. It is also advisable to appraise the client's physical condition always looking for communicable diseases.

The provider needs to do competent assessment to determine if risk of harm exists. If no risk is present, the provider continues with the visit until a change occurs which would require a reassessment. If risk is present, determine if the risk is low, as in an environmental hazard; or moderate, as in an escalating situation threatening potential harm; or high, as in physical harm to person or property is inevitable; and take corrective action as needed. Whenever possible, develop a plan of correction not the frights and always have a safe visit.

Indeed, there is a need for more research in the area of home visit safety regarding individuals with ID and their families and caregivers. 